\title{
Replacement of nuclear protein by histone in pig sperm nuclei during in vitro fertilization
}

\author{
Y. Nakazawa ${ }^{1,2 *}$, A. Shimada ${ }^{3}$, J. Noguchi ${ }^{1}$, I. Domeki ${ }^{2}$, \\ H. Kaneko ${ }^{1}$ and K. Kikuchi ${ }^{1+}$ \\ ${ }^{1}$ Genetic Diversity Department and ${ }^{3}$ Developmental Biology Department, \\ National Institute of Agrobiological Sciences, Kannondai 2-1-2, Tsukuba, Ibaraki 305-8602, \\ Japan; and ${ }^{2}$ Laboratory of Animal Reproduction, Department of Zootechnical Science, \\ Tokyo University of Agriculture, Atsugi, Kanagawa 243-0034, Japan
}

Sperm-specific nuclear protamines are dissociated before decondensation of sperm nuclei during fertilization in pigs. In the present study, replacement of nuclear protein by histone in boar spermatozoa during in vitro fertilization was evaluated by immunohistochemistry using anti-histone antibody. First, the specificity of the antibody used in this study was examined. Immunohistochemistry of the testes and epididymides indicated that somatic nuclei, but not elongated spermatids or maturing spermatozoa, were immunoreactive. Furthermore, immunoreaction was diminished after the antibody had been preincubated with unfractionated histone, indicating that the antibody was specific for the somatic nuclear histone. Immunohistochemistry of serial sections of oocytes, which were matured and co-cultured with boar spermatozoa for 2 to $6 \mathrm{~h}$ indicated that, at 2 to $3 \mathrm{~h}$ after insemination, penetrating sperm nuclei in the condensed state were not immunoreactive. At 4 to $\mathbf{5} \mathrm{h}$ after insemination, some of the condensed sperm nuclei were immunoreactive in part or over the whole area of the nucleus, and all of the decondensing nuclei and male pronuclei were immunoreactive. At $6 \mathrm{~h}$ after insemination, the decondensing sperm nuclei and well-developed male pronuclei were immunoreactive. These results imply that, in pigs, remodelling of sperm nuclear protein from protamine to histone is initiated at the time of sperm penetration, before onset of decondensation and male pronuclear formation.

\section{Introduction}

Fertilization is the first step for embryonic development. Before fertilization, sperm nuclear DNA is packed tightly with strongly basic DNA-binding proteins termed protamines or sperm-specific nuclear proteins. For completion of fertilization, penetrating sperm heads undergo cytological and biochemical changes different from those of oocyte chromatin. In summary, the cytological changes are: fusion of the plasma membrane of acrosome-reacted spermatozoa with the oocyte membrane; decondensation and then recondensation into a mass of chromatin, but not back to the original shape (oval spatulate nuclei); and decondensation of the nuclei again, resulting in male pronucleus formation. During these events, biochemical changes occur to the sperm nuclei. One of the most important events is chromatin remodelling from sperm-specific protamine to somatic nuclear histone. Yanagimachi (1994) suggested that the remodelling requires two steps: first, reduction of $\mathrm{S}-\mathrm{S}$ to $\mathrm{S}-\mathrm{H}$ in protamines, and second, replacement of

* Present address: Organogenesis and Neurogenesis Group, Center for Developmental Biology, RIKEN, Kobe 650-0047, Japan

${ }^{+}$Correspondence

Email: kiku@nias.affrc.go.jp protamines by histones. The ability for $\mathrm{S}-\mathrm{S}$ reduction in ooplasm correlates with the concentration of free thiol glutathione (Perreault et al., 1988; Zirkin et al., 1989). Biochemical analysis of the replacement process of sperm nuclear protein has been carried out using amphibian egg extracts (Lohka and Masui, 1983; Katagiri and Ohsumi, 1994) because these physiological events terminate quickly in the fertilized oocytes of organisms such as sea urchins. However, these events progress slowly in mammalian fertilized oocytes, which might be an advantage for analysis. Shimada et al. (2000) demonstrated that protamine dissociation occurs in fertilized pig oocytes just after sperm penetration but before sperm nuclear decondensation. This observation indicates that replacement by histones is likely to be initiated during the period just after sperm penetration and completed during sperm chromatin decondensation before DNA synthesis in a male pronucleus; this contention differs slightly from conclusions drawn by Nonchev and Tsanev (1990) in mice.

Understanding of the mechanism of sperm nuclear chromatin remodelling during fertilization may be advantageous not only for studies in developmental biology but also for the improvement of the successful rates of using the developing reproductive techniques of intracytoplasmic sperm injection (ICSI) or cloning by nuclear transfer. The 
injected sperm heads should complete their chromatin remodelling in the same way as they do in sperm penetration; however, the relationship between this phenomenon and successful development of sperm-injected oocytes has not yet been determined. In transplanted nuclei (Adenot et al., 2000), interactions may occur between somatic histones brought by the nuclei and oocyte-specific linker histones originating from oocytes, as has been reported in sea urchins (Mandl et al., 1997), frogs (Ohsumi and Katagiri, 1991a) and mice (Tanaka et al., 2001).

In the present study, the correlation between the morphological changes of penetrated sperm nuclei and the dynamics of histone transfer onto sperm nuclei was evaluated by immunohistochemical methods during in vitro fertilization of pig oocytes. Chromatin remodelling during the early period of pig fertilization is discussed in the light of results from this and a previous study (Shimada et al., 2000).

\section{Materials and Methods}

In vitro maturation and fertilization

Pig ovaries were obtained from prepubertal cross-bred gilts (Landrace, Large White and Duroc breeds) at a local abattoir and transported to the laboratory at $35^{\circ} \mathrm{C}$. Cumulusoocyte complexes (COCs) were collected from follicles 2-5 mm in diameter in TCM-199 with Hanks' salts (Gibco, Life Technologies Inc., Grand Island, NY) supplemented with 10\% (v/v) fetal bovine serum (Gibco), 20 mmol Hepes $\mathrm{I}^{-1}$ (Dojindo Laboratories, Kumamoto), 100 iu penicillin G potassium $\mathrm{ml}^{-1}$ (Sigma Chemical Co., St Louis, MO), and $0.1 \mathrm{mg}$ streptomycin sulphate $\mathrm{ml}^{-1}$ (Sigma; Kikuchi et al., 1993). In vitro maturation (IVM) of oocytes was carried out as described by Kikuchi et al. (1999, 2002). In brief, about 50 COCs were cultured in each $500 \mu$ of maturation medium, a modified NCSU-37 solution (Petters and Wells, 1993) containing $10 \%(\mathrm{v} / \mathrm{v})$ pig follicular fluid, $0.6 \mathrm{mmol}$ cysteine $\mathrm{I}^{-1}, 0.05 \mathrm{mmol} \beta$-mercaptoethanol $\mathrm{I}^{-1}, 1 \mathrm{mmol}$ dibutyryl cAMP I-1 (dbcAMP; Sigma), 10 iu eCG $\mathrm{ml}^{-1}$ (PMS 1000 iu; Nihon Zenyaku Kogyo, Koriyama), and 10 iu hCG $\mathrm{ml}^{-1}$ (Puberogen 500 unit; Sankyo, Tokyo), in four-well dishes (Nunclon Multidishes, Nalge Nunc International, Roskilde) for 20 h. COCs were subsequently cultured in the maturation medium without dbcAMP and hormones for $24 \mathrm{~h}$. The maturation culture was carried out under $5 \% \mathrm{CO}_{2}$ in air at $39^{\circ} \mathrm{C}$. After maturation culture, oocytes with expanded cumulus cells were treated briefly with 150 iu hyaluronidase $\mathrm{ml}^{-1}$ and denuded of cumulus cells by gentle pipetting. The denuded oocytes with the first polar body were harvested under a stereomicroscope and served as matured oocytes for in vitro fertilization (IVF). IVF was carried out as described by Kikuchi et al. (1995). Epididymal spermatozoa from a Landrace boar were frozen (Kikuchi et al., 1998; Ikeda et al., 2002). After thawing and pre-incubation for $1 \mathrm{~h}$ at $37^{\circ} \mathrm{C}$ in TCM-199 (with Earle's salts; Gibco) adjusted to pH 7.8 (Nagai et al., 1988; Ikeda et al., 2002), the spermatozoa were diluted once with Bracket and Oliphant solution (Bracket and Oliphant, 1975) supplemented with $5 \mathrm{mmol}$ caffeine $\mathrm{I}^{-1}$ (Sigma) and $100 \mathrm{mg}$ casein phosphopeptide $\mathrm{ml}^{-1}$ (Meiji Seika Kaisha Ltd, Tokyo), and a portion of the diluted spermatozoa $(10 \mu \mathrm{l})$ was transferred to $90 \mu \mathrm{l}$ of fertilization medium containing ten matured oocytes. The final sperm concentration was $1 \times 10^{5}$ cells ml- $^{-1}$.

\section{Examination of fertilization status}

After co-incubation of gametes for 2, 3, 4, 5, 6, 8 or $10 \mathrm{~h}$, some oocytes were freed from the attached spermatozoa, whole-mounted on to a glass slide, fixed in acetic alcohol $(1: 3)$, stained with $1 \%(\mathrm{w} / \mathrm{v})$ aceto-orcein solution, and examined under a phase-contrast microscope.

\section{Evaluation of specificity of anti-histone antibody}

Before examination in samples from fertilized oocytes, the specificity of anti-histone antibody (monoclonal antihistone H1; Leinco Technologies, Inc., St Louis, MO) used for immunohistochemistry was evaluated. Immunohistochemical approaches were conducted essentially as described by Noguchi et al. (1997). Tissue samples of testis and epididymis from adult Landrace boar were fixed with Methacarn solution (methanol-chloroform-acetic acid, $6: 3: 1$ by volume), and dehydrated with a series of alcohols, and embedded in paraffin wax. Sections were cut at $4 \mu \mathrm{m}$, deparaffinized, treated with $8 \mathrm{~mol}_{\text {urea }}{ }^{-1}$ for $30 \mathrm{~min}$ for generation of antigen, and then all sections were incubated in $0.5 \%(\mathrm{w} / \mathrm{v})$ periodic acid to block endogenous peroxidase. After blocking with $0.5 \%(\mathrm{w} / \mathrm{v})$ casein Tris-buffered saline (TBS), the sections were incubated overnight at $4{ }^{\circ} \mathrm{C}$ with the anti-histone antibody at a dilution of $1: 5$ in casein TBS. The Elite ABC kit (Vector Laboratories Inc., Burlingame, CA) with diaminobenzidine was used to visualize the reaction.

The specificity of the antibody was also examined by testing whether immunopositive reactions were diminished by the use of immunoabsorbed antiserum, which was prepared as follows. The antibody at a dilution of 1:40 in TBS was mixed with unfractionated whole histone (Type II-A from calf thymus; Sigma) to a final concentration of $0-2560 \mu \mathrm{g} \mathrm{ml}^{-1}$. After incubation at $37^{\circ} \mathrm{C}$ for $1 \mathrm{~h}$ and then at $4^{\circ} \mathrm{C}$ overnight, the mixture was centrifuged at $10000 \mathrm{~g}$ for $1 \mathrm{~h}$ at $4^{\circ} \mathrm{C}$. The supernatant was used for immunohistochemical staining at the same dilution as untreated antiserum.

Detection ability was also determined in oocytes. Maturing oocytes cultured for $24 \mathrm{~h}$, matured oocytes cultured for $44 \mathrm{~h}$ and fertilized oocytes at $2 \mathrm{~h}$ after insemination were fixed in $3 \%(\mathrm{w} / \mathrm{v})$ paraformaldehyde and processed for immunohistochemical evaluation as described by Shimada et al. (2000). In brief, the fixed oocytes were embedded in 4\% agar (Difco Laboratories, Detroit, MI), and then the agar containing oocytes was dehydrated and embedded in paraffin wax. After deparaffinization, serial sections ( $3 \mu \mathrm{m}$ in thickness) were processed for immunohistochemistry. The procedure for immunohistochemistry 

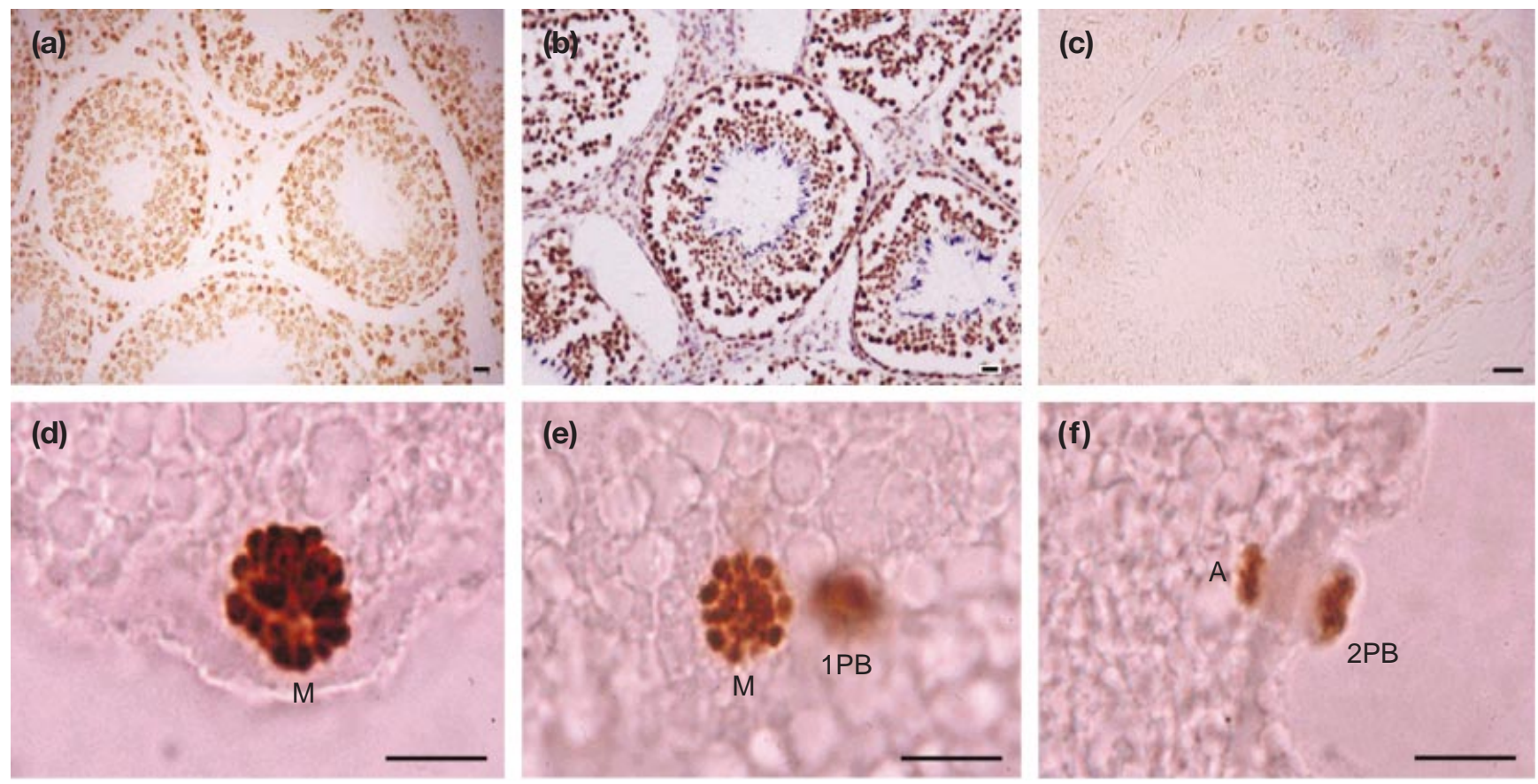

Fig. 1. (a) Immunohistochemistry with anti-histone $\mathrm{H} 1$ antibody of adult boar testis. (b) Immunohistochemistry followed by counterstaining with haematoxylin. Nuclei of elongated spermatids in the seminiferous epithelium showed staining with haematoxylin but not with diaminobenzidine, indicating dissociation of histone $\mathrm{H} 1$, whereas spermatogonia, spermatocytes and round spermatids associated with histone $\mathrm{H} 1$ showed staining with diaminobenzidine. (c) Immunohistochemistry of boar testis with anti-histone antibody preincubated with unfractionated histones $\left(2560 \mu \mathrm{g} \mathrm{m}^{-1}\right)$. Most of the positive reactions in nuclei of germ cells such as spermatogonia, spermatocytes and round spermatids, and those of the interstitial cells are no longer present. (d-f) Immunohistochemistry applied in pig oocytes. (d) Maturing (unfertilized) oocyte at metaphase I. (e) Matured (unfertilized) oocyte at metaphase-II, and (f) fertilized oocytes at anaphase II emitting the second polar body. Oocyte chromatin at metaphase (M) or anaphase (A) stages and both first (1PB; out of focus) and second (2PB) polar bodies were immunoreactive. Scale bars represent $10 \mu \mathrm{m}$.

of oocytes was the same as described above, except for autoclaving at $121^{\circ} \mathrm{C}$ for $5-15 \mathrm{~min}$ in 0.1 mol citric acid $\mathrm{I}^{-1}$ $(\mathrm{pH} 6.0)$ and blocking with $1.5 \%$ normal goat serum (Vector).

\section{Immunohistochemical evaluation of IVF oocytes}

The IVF oocytes at 2, 3, 4, 5, 6, 8 or $10 \mathrm{~h}$ after insemination were fixed and processed for immunohistochemistry as described above. After immunohistochemical staining, serial sections were counterstained with haematoxylin. Morphological changes of penetrating sperm nuclei and immunoreactivity with anti-histone antibody were evaluated.

\section{Results}

\section{Specificity of the anti-histone antibody in pig tissues}

Immunohistochemical staining of boar testis showed specific reaction in nuclei of interstitial cells or those of germ cells such as spermatogonia, spermatocytes and round spermatids in seminiferous epithelium but not in nuclei of elongated spermatids (Fig. 1a). When immunostaining was followed by staining with haematoxylin, nuclei of elongated spermatids located in the luminal area were stained with haematoxylin alone, whereas the other cells in the seminiferous epithelium were dark brown, indicating staining by both diaminobenzidine and haematoxylin (Fig. 1b). No positive reaction was detected in the matured epididymal spermatozoa (data not shown). An immunoabsorbance test with the antibody preincubated with unfractionated histone was conducted to confirm the specificity of the staining. Nuclei of germ cells such as spermatogonia, spermatocytes and round spermatids were positive without immunoabsorbance (Fig. 1a); however, most of the positive reactions diminished after preincubation with histone, as the histone concentration increased (Fig. 1c). The ability of the antibody to detect histone in maturing, matured and activated oocytes was also examined. Metaphase I and II and anaphase II chromosomes (Fig. 1d-f) and nuclei of first and second polar bodies (Fig. 1e,f) were detected by immunohistochemistry with this antibody.

\section{Sequential nuclear changes of inseminated oocytes and penetrating spermatozoa}

Sequential changes of penetrating sperm nuclei were observed in whole-mount preparations of inseminated 


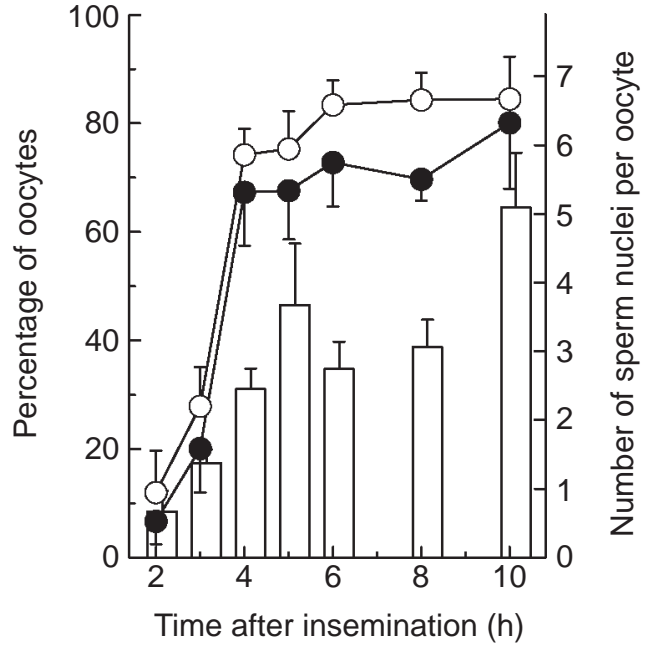

Fig. 2. Rate of sperm penetration $(\bigcirc)$, rate of polyspermy $(\bullet)$ and number of spermatozoa per oocyte (histogram) of pig oocytes fertilized in vitro. Oocytes matured in vitro were inseminated and fixed in whole mount preparation at 2, 3, 4, 5, 6, 8 or $10 \mathrm{~h}$ after insemination. The oocytes were stained with $1 \%(\mathrm{w} / \mathrm{v})$ acetoorcein and examined by phase-contrast microscopy. Means \pm SEM are presented. At least three replicated trials were performed and a total of 90-153 oocytes were examined for each category.

oocytes. The rates of sperm penetration, polyspermy and the number of penetrated spermatozoa per oocyte are shown (Fig. 2). Sperm penetration was observed in $>10 \%$ of the oocytes at $2 \mathrm{~h}$ after insemination. At $4-10 \mathrm{~h}$, about $80 \%$ of inseminated oocytes were penetrated, and most of them showed polyspermic fertilization, indicating increased numbers of penetrating spermatozoa. Inseminated oocytes were activated to be at anaphase II at $2 \mathrm{~h}$, proceeded to telophase II at about $4 \mathrm{~h}$, and began to form a female pronucleus from $4 \mathrm{~h}$ after insemination (Fig. 3a). Whole-mount preparations of inseminated oocytes also showed the detail of morphological changes of sperm nuclei (Fig. 3b). Sperm entry was first observed at $2 \mathrm{~h}$ after insemination, when sperm nuclei were in a condensed state. Decondensation of the nuclei started from $3 \mathrm{~h}$ and continued to $10 \mathrm{~h}$ after insemination. A male pronucleus was first observed to form at $4 \mathrm{~h}$ after insemination, but was small. Well-developed pronuclei with a nucleolus appeared from $6 \mathrm{~h}$ after insemination. This development almost matches female pronuclear development.

\section{Histone status in penetrated sperm nuclei}

Examination of sectioned oocytes under light microscopy enabled visualization of (i) condensed sperm nuclei of the original shape, (ii) decondensing (including recondensing) sperm nuclei, and (iii) male and female pronuclei. Immunoreactivity in the sperm nuclei after penetration is shown (Fig. 4). Three types of condensed sperm nuclei were identified by the pattern of staining (Fig. $4 a-c$ ): (i) histone

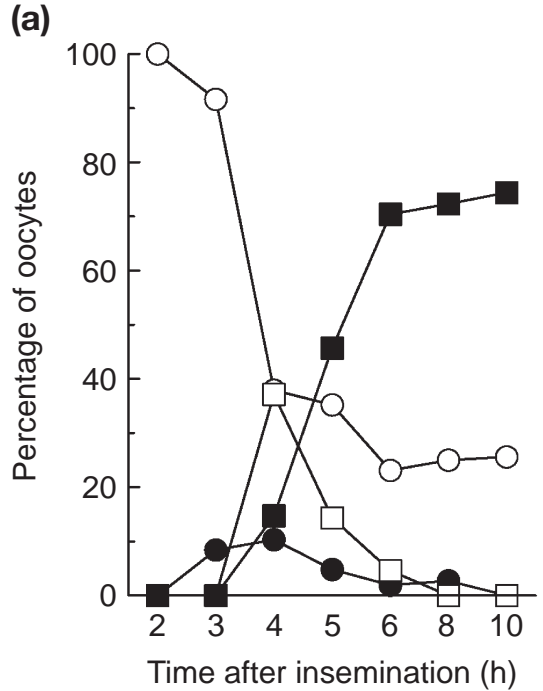

(b)

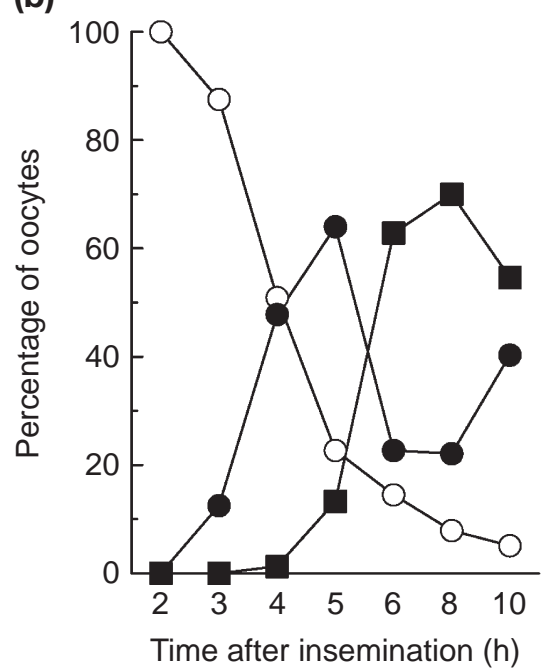

Fig. 3. (a) Oocyte nuclear status and (b) morphological changes of penetrating sperm nuclei of pig oocytes fertilized in vitro. (a) Sperm penetration was initiated at $2 \mathrm{~h}$ after insemination; however, oocyte chromatin was arrested at metaphase II $(\bigcirc)$. Oocytes were activated and their nuclear status proceeded to anaphase II (O) at $3 \mathrm{~h}$. After reaching telophase II ( $\square$ ), the female pronucleus began to develop from $4 \mathrm{~h}$. Female pronucleus formation with second polar body (ם) occurred in more than $70 \%$ of the oocytes. (b) Sperm penetration was first observed at $2 \mathrm{~h}$ after insemination, and at this stage all of the spermatozoa were condensed $(\bigcirc)$. Decondensing nuclei $(\mathbf{)})$ began to be observed after $3 \mathrm{~h}$ and male pronuclei ( after $4 \mathrm{~h}$. Male pronuclear formation occurred in about $60 \%$ of the inseminated oocytes. At least three replicated trials were performed and a total of 90-164 oocytes were examined for each category.

not associated with the sperm nucleus: no diaminobenzidine signal observed and sperm nuclei stained with haematoxylin only; (ii) histone associated with part of the nucleus: part of the area of sperm nuclei stained with diaminobenzidine and the remaining area stained with haematoxylin; and (iii) histone associated with the entire nucleus: diamino- 

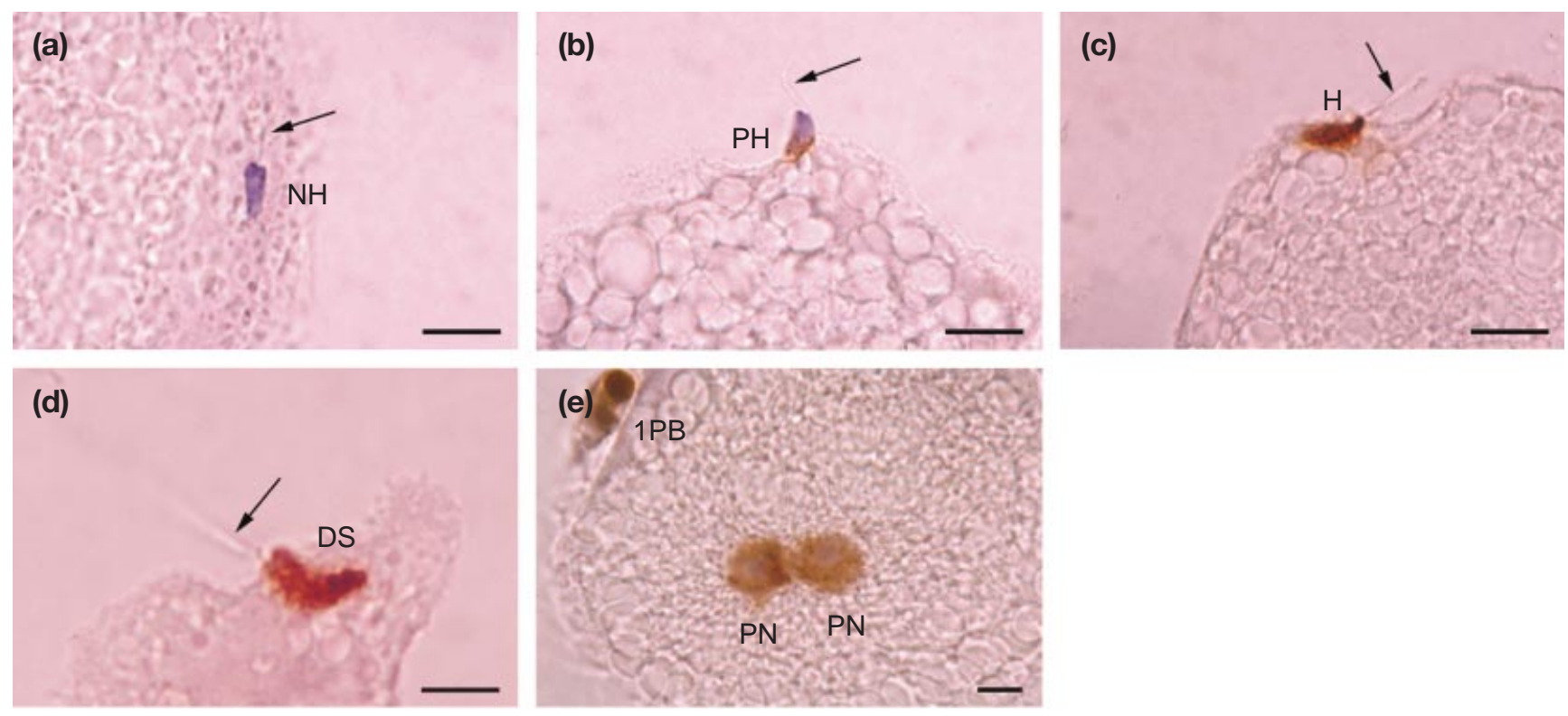

Fig. 4. Immunohistochemistry with anti-histone $\mathrm{H} 1$ antibody followed by staining with haematoxylin allowing histone $\mathrm{H} 1$-associated sperm nuclei from non-associated nuclei to be distinguished in fertilized pig oocytes. Immunoreaction resulted in dark-brown staining with both diaminobenzidine and haematoxylin, whereas chromatin without histone $\mathrm{H} 1$ association resulted in blue staining with haematoxylin. (a) A condensed sperm nucleus at $3 \mathrm{~h}$ after insemination showed staining with haematoxylin alone ( $\mathrm{NH}$ ), indicating no association of histone $\mathrm{H} 1$ with the sperm nucleus. (b) A part of a condensed sperm nucleus at $4 \mathrm{~h}$ after insemination showed staining with haematoxylin and the remaining area showed staining with diaminobenzidine $(\mathrm{PH})$. This finding indicates that some of the condensed sperm nuclei had associated with histone H1. (c) A condensed sperm nucleus showed staining with diaminobenzidine (H) at $4 \mathrm{~h}$ after insemination. (d) A sperm nucleus in an oocyte showing heavy polyspermy. Sperm nuclei were highly decondensed (DS), resulting in the broad staining with diaminobenzidine at $5 \mathrm{~h}$ after insemination. (e) Male and female pronuclei (PN) showed staining with diaminobenzidine at $6 \mathrm{~h}$ after insemination. They were not distinguishable from each other. The first polar body (1PB) was immunoreactive. Arrows indicate sperm tails. Scale bars represent $10 \mu \mathrm{m}$.

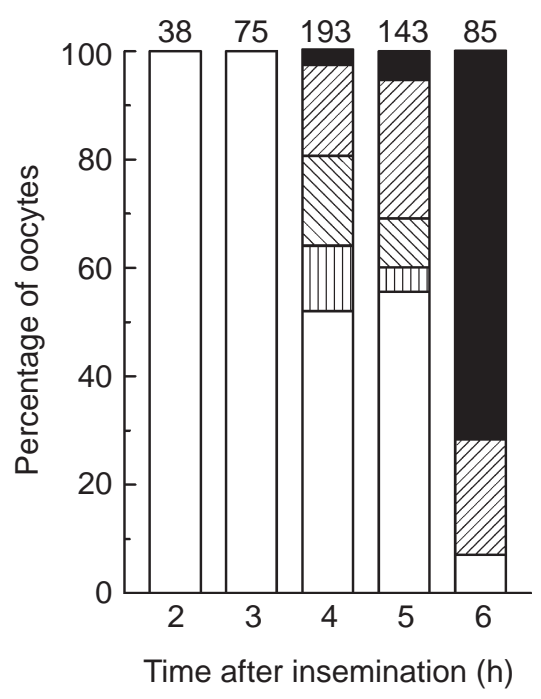

Fig. 5. Relationship between morphological changes of penetrating pig sperm nuclei and their immunoreactivity with anti-histone $\mathrm{H} 1$ antibody. At $2-3 \mathrm{~h}$ after insemination, all of the penetrated sperm nuclei were condensed and showed staining only with haematoxylin. At 4-5 h, some condensed sperm nuclei were immunoreactive in part of (haematoxylin and diaminobenzidine) or over the whole area (diaminobenzidine) of the nuclei. Up to $6 \mathrm{~h}$, all of the decondensing sperm nuclei and male pronuclei were benzidine signal observed all over the sperm nuclei and haematoxylin staining not visible. All the decondensing sperm nuclei (Fig. 4d) and both male and female pronuclei (Fig. 4e) were stained with diaminobenzidine and the signal from haematoxylin only was not detected. Because distinguishing the male from the female pronucleus was impossible in the sections, one was subtracted as a female pronucleus in the case of multiple pronuclear formation. In the case of polyspermy, all the decondensing sperm nuclei and pronuclei were equally labelled with diaminobenzidine.

The sequential changes of penetrated spermatozoa according to their morphological status and their immunoreactions are summarized (Fig. 5). Before $4 \mathrm{~h}$ after insemination, all penetrated sperm nuclei were condensed, and immunoreactivity showed that histone not associated with the sperm nucleus. At $4-5 \mathrm{~h}$ after insemination, about $80 \%$ of penetrated sperm nuclei were condensed, but all three types of immunohistochemical reactivity ('histone not associated with the sperm nucleus', 'histone associated with

immunoreactive (diaminobenzidine). $\mathbf{\square}$ : Pronucleus, diaminobenzidine; 四: decondensing, diaminobenzidine; $\mathbb{\mathbb { N }}$ : condensed, diaminobenzidine; $\square$ : condensed, haematoxylin and diaminobenzidine; and $\square$ : condensed, haematoxylin. Numbers on the bars represent the total number of sperm nuclei used for analysis. 


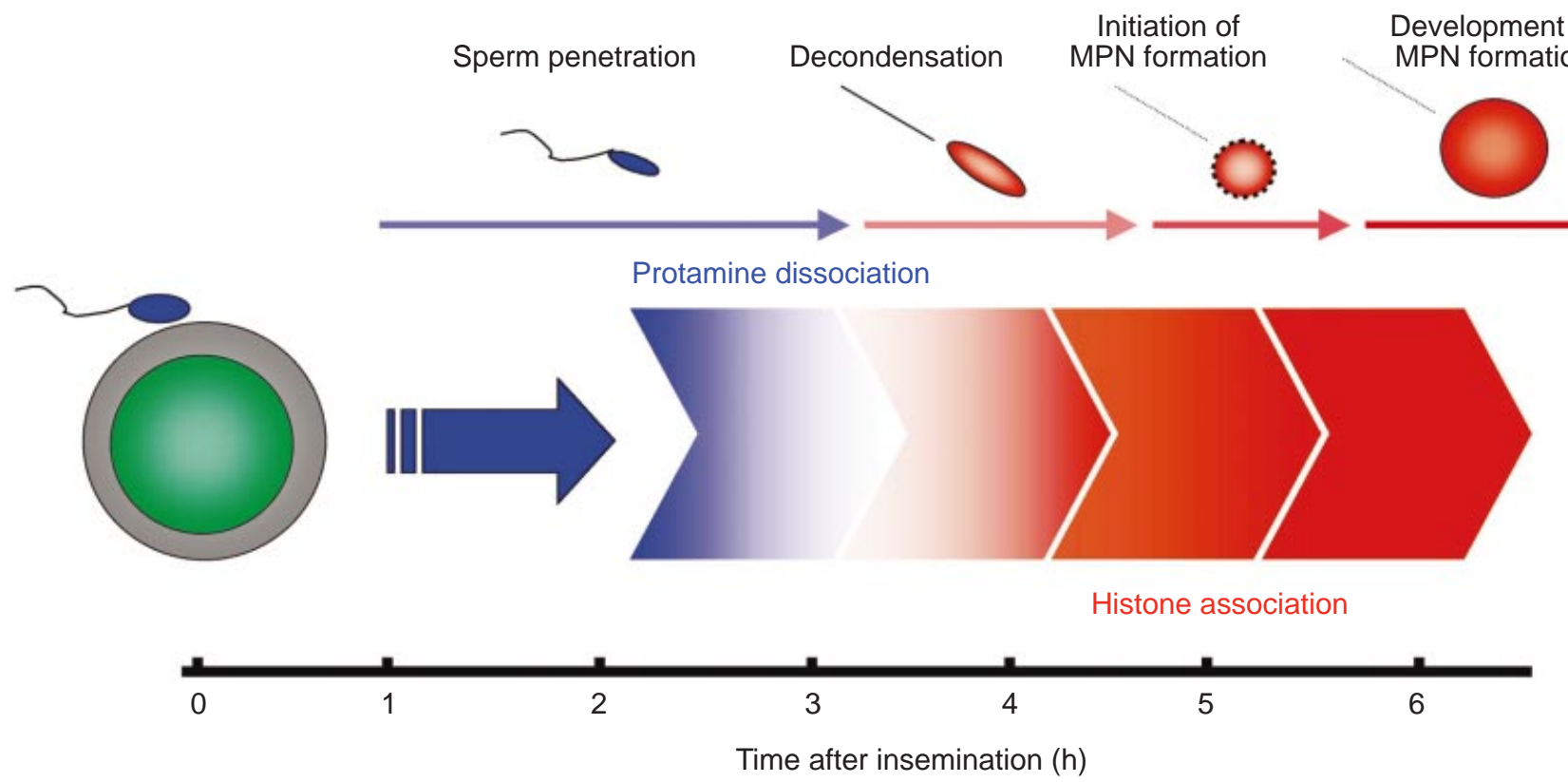

Fig. 6. Schematic diagram of remodelling of boar sperm nuclear protein from protamine to histone in relation to changes in sperm nuclear morphology. Protamine dissociation occurs at $2-3 \mathrm{~h}$ and histone association occurs until $4 \mathrm{~h}$ after insemination, before decondensation or male pronucleus (MPN) formation.

part of the nucleus' and 'histone associated with the entire nucleus') were observed. Decondensing sperm nuclei and pronuclei, which were judged to have developed from sperm nuclei, were also immunoreactive and considered to show 'histone associated with the entire nucleus'. At $6 \mathrm{~h}$ after insemination, most of the penetrated sperm nuclei were decondensing and developing into male pronuclei; these were considered to show 'histone associated with the entire nucleus'. A small percentage of condensed sperm nuclei without immunoreaction was also observed.

\section{Discussion}

During fertilization, protamines dissociate from penetrating boar sperm nuclei that are still condensed at $2-3 \mathrm{~h}$ after insemination (Shimada et al., 2000), at which time the spermatozoa begin to penetrate the oocytes. The results of the present study clearly indicate that some of the condensed sperm nuclei at $4-5 \mathrm{~h}$ after insemination are immunoreactive and that all of the decondensing nuclei and pronuclei are immunoreactive after $4 \mathrm{~h}$. On the basis of these results, it is suggested that protamines dissociate from the boar nuclei just after penetration and, just after the protamine dissociation, histones began to associate in the nuclei before decondensation or formation of the pronucleus (Fig. 6). In mice, although the idea that protamine loss occurs as histones appear in the well-developed male pronucleus is accepted (Nonchev and Tsanev, 1990), it can be concluded that sperm basic proteins are retained in the decondensed nuclei (Rodman et al., 1981). As the timing of the remodelling in mice (whether in condensed or decondensed sperm nuclei) is not yet known, the present study in pigs is the first to have used immunohistochemistry to clarify the remodelling of penetrated sperm nuclei. In other mammalian species, further attention should be paid to the mechanism of remodelling of the nuclei after the sperm penetration.

After sperm penetration, protamine dissociation occurs and histone association and then somatic nucleosome reconstruction are observed (Yanagimachi, 1994). In frogs, remodelling of nuclear protein from protamine to histone occurs just after penetration (Ohsumi and Katagiri, 1991b). In mammals, especially in domestic animals such as pigs, the remodelling processes are not yet well understood. However, the observations in the previous (Shimada et al., 2000) and present studies indicate that the dissociation of protamine and association of histone in penetrating sperm nuclei occur just after sperm penetration and before decondensation of nuclei, indicating that the nucleosome structure in sperm chromatin is reconstructed at the initial stage of fertilization and is similar to that of oocyte chromatin. The equality in the molecular structure between male and female chromatin appears to be important for the synchronization of decondensation and subsequent pronucleus formation between male and female chromatin, and may affect pronuclear functions such as DNA replication or transcriptional activity. In fact, synchronization has been detected between formation of male and female pronuclei in the present study, and in DNA replication within the pronuclei in fertilized mammalian oocytes (hamster: Naish et al., 1987; pig: Laurincik et al., 1995). Further functional analysis, for example examining transcriptional activity, of the pronuclei is expected to produce similar results in pigs as it has in mice (Aoki and Schultz, 1999). 
In general, histones are well-conserved; however, many subtypes of linker histone (H1) are known (van Holde, 1988). Oocyte- or embryo-specific H1 subtypes, which can be distinguished by immunological approaches, have been reported, in particular, in sea urchins and frogs (Pehrson and Cohen, 1984; Smith et al., 1988). Somatic H1 subtypes have not been detected in early embryos of mammals or non-mammalian animals. In mice, somatic $\mathrm{H} 1$ subtypes are not detected in germinal vesicles, the nuclei of polar bodies or the nuclei of one- or two-cell-stage embryos, but are detected in the nuclei of embryos beyond the four-cell stage (Clarke et al., 1992). In cattle, somatic H1 assembly is similar to that found in mice (Smith et al., 1995). Tanaka et al. (2001) reported evidence of a mouse oocyte- or embryospecific linker histone subtype, named H1oo. H1oo has been detected in germinal vesicles, metaphase II chromosomes, the nuclei of polar bodies, pronuclei, and nuclei at the two-cell stage, and shows similarity to the oocytespecific linker histone (B4/H1M) of frogs (Dworkin-Rastl et al., 1994) and the cleavage stage-specific linker histone (cs-H1) of sea urchins (Poccia et al., 1981). The antibody used in the present study detected somatic histone in pig testes and epididymides and its specificity was confirmed by an immunoabsorbance test. Penetrated sperm nuclei, oocyte chromatin in both metaphase to anaphase chromosomes, the nuclei of polar bodies and the female pronucleus were all shown to be immunoreactive with the antibody. This finding indicates that the anti-histone antibody used in the present study might detect both oocyte-specific $\mathrm{H} 1$ and somatic $\mathrm{H} 1$ subtypes in pigs; however, there is no evidence for a pig oocyte-specific $\mathrm{H} 1$ subtype. Further investigation is necessary to determine the $\mathrm{H} 1$ subtype involved in the remodelling during pig fertilization.

In mammals, little is known about the oocyte factors that are responsible for protamine-histone exchange and nuclear decondensation during fertilization. Cytoplasmic proteins called nuclear chaperones mediate protaminehistone exchange and decondensation of sperm nuclei in oocytes (for a review, see Philpott et al., 2000). One of the well-studied nuclear chaperones is nucleoplasmin. In amphibians, nucleoplasmin removes protamine or spermspecific basic proteins, while it deposits an $\mathrm{H} 2 \mathrm{~A}-\mathrm{H} 2 \mathrm{~B}$ dimer (Ohsumi and Katagiri, 1991b; Philpott et al., 1991; Philpott and Leno, 1992). Itoh et al. (1993) demonstrated that remodelling of human sperm chromatin is observed in amphibian egg extracts and is mediated by nucleoplasmin from egg extracts. Furthermore, there is a nuclear factor in interphase egg nucleus that affects sperm decondensation and is released into the ooplasm as oocytes mature (Maeda et al., 1998). These results indicate the presence of nucleoplasmin-like substances in mammalian oocytes. Members of the nucleoplasmin family of nuclear chaperones have been isolated in mice (MacArthur and Shackleford, 1997) and humans (Shackleford et al., 2001). However, there is no firm evidence as yet for nucleoplasmin-like chaperone molecules functioning in protamine-histone exchange of mammalian sperm. In addition, the mechanism for transfer of histone $\mathrm{H} 1$ to sperm nuclei has not yet been identified even in amphibians. Further attempts to clarify the mechanism are needed.

In conclusion, the results of the present study indicate that somatic histone or its variant associates with boar sperm nuclei just after their penetration and after dissociation of protamine from the nuclei.

The authors would like to thank T. Aoki, E. Yamauchi, M. Irie and M. Sakurai for technical assistance.

\section{References}

Adenot PG, Campion E, Legouy E, Allis CD, Dimitrov S, Renard JP and Thompson EM (2000) Somatic linker histone H1 is present throughout mouse embryogenesis and is not replaced by variant $\mathrm{H} 1^{\circ}$ Journal of Cell Science 113 2897-2907

Aoki F and Schultz RM (1999) DNA replication in the 1-cell mouse embryo: stimulatory effect of histone acetylation Zygote 7 165-172

Bracket BG and Oliphant G (1975) Capacitation of rabbit spermatozoa in vitro. Biology of Reproduction 12 260-274

Clarke HJ, Oblin C and Bustin M (1992) Developmental regulation of chromatin composition during mouse embryogenesis: somatic histone H1 is first detectable at the 4-cell stage Development 115 791-799

Dworkin-Rastl E, Kandolf H and Smith RC (1994) The maternal histone H1 variant, $\mathrm{H} 1 \mathrm{M}$ (B4 protein), is the predominant $\mathrm{H} 1$ histone in Xenopus pregastural embryos Developmental Biology 161 425-439

Ikeda H, Kikuchi K, Noguchi J, Takeda H, Shimada A, Mizokami T and Kaneko H (2002) Effect of preincubation of cryopreserved porcine epididymal sperm Theriogenology 57 1309-1318

Itoh T, Ohsumi K and Katagiri C (1993) Remodeling of human sperm chromatin mediated by nucleoplasmin from amphibian eggs Development Growth and Differentiation 35 59-66

Katagiri C and Ohsumi K (1994) Remodeling of sperm chromatin induced in egg extracts of amphibians International Journal of Developmental Biology 38 209-216

Kikuchi K, Nagai T, Motlik J, Shioya Y and Izaike Y (1993) Effect of follicle cells on in vitro fertilization of pig follicular oocytes Theriogenology 39 593-599

Kikuchi K, Naito K, Dean FP, Izaike Y and Toyoda Y (1995) Histone H1 kinase activity during in vitro fertilization of pig follicular oocytes matured in vitro. Theriogenology 43 523-532

Kikuchi K, Nagai T, Kashiwazaki N, Ikeda H, Noguchi J, Shimada A, Soloy E and Kaneko H (1998) Cryopreservation and ensuing in vitro fertilization ability of boar spermatozoa from epididymides stored at $4{ }^{\circ} \mathrm{C}$ Theriogenology 50 615-623

Kikuchi K, Kashiwazaki N, Noguchi J, Shimada A, Takahashi R, Hirabayashi M, Shino M, Ueda M and Kaneko H (1999) Developmental competence, after transfer to recipients, of porcine oocytes matured, fertilized, and cultured in vitro. Biology of Reproduction 60 336-340

Kikuchi K, Onishi A, Kashiwazaki N, Iwamoto M, Noguchi J, Kaneko H, Akita T and Nagai T (2002) Successful piglet production after transfer of blastocysts produced by a modified in vitro system Biology of Reproduction 66 1033-1041

Laurincik J, Hyttel P and Kopecny V (1995) DNA synthesis and pronucleus development in pig zygotes obtained in vivo: an autoradiographic and ultrastructural study Molecular Reproduction and Development 40 325-332

Lohka MJ and Masui Y (1983) Formation in vitro of sperm pronuclei and mitotic chromosomes induced by amphibian ooplasmic components Science 220 719-721

MacArthur CA and Shackleford GM (1997) Npm3: a novel, widely expressed gene encoding a protein related to the molecular chaperones nucleoplasmin and nucleophosmin Genomics 42 137-140

Maeda Y, Yanagimachi H, Tateno H, Usui N and Yanagimachi R (1998) Decondensation of the mouse sperm nucleus within the interphase nucleus Zygote 6 39-45 
Mandl B, Brandt WF, Superti-Furga G, Graninger PG, Birnstiel ML and Busslinger M (1997) The five cleavage-stage (CS) histones of the sea urchin are encoded by a maternally expressed family of replacement histone genes: functional equivalence of the $\mathrm{CS} \mathrm{H} 1$ and frog $\mathrm{H} 1 \mathrm{M}$ (B4) proteins Molecular and Cellular Biology 17 1189-1200

Nagai T, Takahashi T, Masuda H, Shioya Y, Kuwayama M, Fukushima M, Iwasaki S and Hanada A (1988) In vitro fertilization of pig oocytes by frozen boar spermatozoa Journal of Reproduction and Fertility 84 585-591

Naish SJ, Perreault SD, Foehner A and Zirkin BR (1987) DNA synthesis in the fertilizing hamster sperm nucleus: sperm template availability and egg cytoplasmic control Biology of Reproduction 36 245-253

Noguchi J, Hikono H, Sato S, Watanabe G, Taya K, Sasamoto S and Hasegawa Y (1997) Ontogeny of inhibin secretion in the rat testis: secretion of inhibin-related proteins from fetal Leydig cells and of bioactive inhibin from Sertoli cells Journal of Endocrinology 155 27-34

Nonchev S and Tsanev R (1990) Protamine-histone replacement and DNA replication in the male mouse pronucleus Molecular Reproduction and Development 25 72-76

Ohsumi K and Katagiri C (1991a) Occurrence of H1 subtypes specific to pronuclei and cleavage-stage cell nuclei of anuran amphibians Developmental Biology 147 110-120

Ohsumi K and Katagiri C (1991b) Characterization of the ooplasmic factor inducing decondensation of and protamine removal from toad sperm nuclei: involvement of nucleoplasmin Developmental Biology 148 295-305

Pehrson JR and Cohen LH (1984) Embryonal histone H1 subtypes of the sea urchin Strogylocentrotus purpuratus: purification, characterization, and immunological comparison with $\mathrm{H} 1$ subtypes of the adult Biochemistry 23 6761-6764

Perreault SD, Barbee RR and Slott VL (1988) Importance of glutathione in the acquisition and maintenance of sperm nuclear decondensing activity in maturing hamster oocytes Developmental Biology 125 181-186

Petters RM and Wells KD (1993) Culture of pig embryos Journal of Reproduction and Fertility Supplement 48 61-73

Philpott A and Leno GH (1992) Nucleoplasmin remodels sperm chromatin in Xenopus egg extracts Cel/ 67 759-767

Philpott A, Leno GH and Laskey RA (1991) Sperm decondensation in Xenopus egg cytoplasm is mediated by nucleoplasmin Cell 65 569-575

Philpott A, Krude T and Laskey RA (2000) Nuclear chaperones Seminars in Cell and Developmental Biology 11 7-14
Poccia D, Salik J and Krystal G (1981) Transitions in histone variants of the male pronucleus following fertilization and evidence for a maternal store of cleavage-stage histones in the sea urchin egg Developmental Biology 82 287-296

Rodman TC, Pruslin FH, Hoffman HP and Allfrey VG (1981) Turnover of basic chromosomal proteins in fertilized egg: a cytoimmunochemical study of events in vivo. Journal of Cell Biology 90 351-361

Shackleford GM, Ganguly A and MacArthur CA (2001) Cloning, expression and nuclear localization of human NPM3, a member of the nucleophosmin/nucleoplasmin family of nuclear chaperones BioMed Central Genomics 28

Shimada A, Kikuchi K, Noguchi J, Akama K, Nakano M and Kaneko H (2000) Protamine dissociation before decondensation of sperm nuclei during in vitro fertilization of pig oocytes Journal of Reproduction and Fertility 120 247-256

Smith L, Meirelles FV, Bustin M and Clarke HJ (1995) Assembly of somatic histone $\mathrm{H} 1$ onto chromatin during bovine early embryogenesis Journal of Experimental Zoology 273 317-326

Smith RC, Dworkin-Rastl E and Dworkin MB (1988) Expression of H1-like protein is restricted to early Xenopus development Genes and Development 10 1284-1285

Tanaka M, Hennebold JD, Macfariane J and Adashi EY (2001) A mammalian oocyte-specific linker histone gene H1oo: homology with the genes for the oocyte-specific cleavage stage histone ( $c s-H 1)$ of sea urchin and the $B 4 / \mathrm{H} 1 \mathrm{M}$ histone of the frog Development 128 655-664

van Holde KE (1988) Chromatin pp 91-111. Springer-Verlag, New York

Yanagimachi R (1994) Mammalian fertilization. In The Physiology of Reproduction 2nd Edn pp 189-317 Eds E Knobil and JD Neill. Raven Press Ltd, New York

Zirkin BR, Perreault SD and Naish SJ (1989) Formation and function of the male pronucleus during mammalian fertilization. In The Molecular Biology of Fertilization pp 91-114 Academic Press, San Diego, CA

Received 26 March 2002.

First decision 17 May 2002.

Revised manuscript received 5 June 2002.

Accepted 12 July 2002. 
